\section{A better value for Earth's mass}

SIR - The uncertainty in the Earth's mass was recently discussed (Nature 302, 11; 1983) but unfortunately without considering the now most accurate value of the gravitational constant

$G=(6.6726 \pm 0.0005) 10^{-11} \mathrm{~m}^{3} \mathrm{~s}^{-2} \mathrm{~kg}^{-1}$

obtained by G.G. Luther and W.R. Towler at the US National Bureau of Standards (see Phys. Rev. Lett. 48, 121; 1982). By using the new value, uncertain by 7.5 parts in 100,000 , and the product

$G M_{\oplus}=3.98600510^{14} \mathrm{~m}^{3} \mathrm{~s}^{-2}$

known from satellite observations with the uncertainty of 1 part in $10^{7}$, we get the new value of the Earth's mass

$$
M_{\oplus}=(5.9737 \pm 0.0004) 10^{24} \mathrm{~kg}
$$

This is more accurate by an order of magnitude than the quoted value of $(5.974 \pm$ $0.004) 10^{24} \mathrm{~kg}$.

One interesting point arises from the measurements of Luther and Towler, who measured the period of a torsional pendulum suspended in an evacuated cylin- drical chamber when two large spheres of tungsten placed in the air outside the chamber were brought near to the pendulum. Then the spheres were removed and the period measured again.

Luther and Towler applied a correction of approximately 1 part in $10^{4}$ to the measured value of $G$ to allow for the fact that when the spheres of tungsten were removed, they were replaced by spheres of air and, consequently, that the change in the force was appropriate to the differences between the mass of tungsten and the mass of air (personal communication). In fact, it can be shown (see Z.H., Proc. 6th and 7th Confs. Czech. Physicists, Ostrava 1979 and Prague 1981) that the gravitational force between homogenous spherical objects immersed in an infinite homogenous fluid can be obtained by substituting for the masses in the usual newtonian expression the difference between the mass of the object and that of the fluid displaced.

Corrections for the effects of buoyancy are of course routinely made in accurate measurements of terrestrial gravitational attraction. More generally, allowing for the displacement of fluid implies that while objects both more or less dense than the immersing fluid will attract each other, those with opposite buoyancy will repel each other. The effect of the medium on the gravitational interaction between objects is thus analogous to that of a dielectric medium on the electrostatic interaction of charges (although the signs are different).

In the well-known measurement of $G$ by Cavendish (1798), in which the mutual attractions of pairs of large and small spheres of lead were measured in air, the buoyancy correction would have amounted to two parts in 10,000 irrelevant compared with the errors of measurement leading to an uncertainty of $1 \%$ in the value of $G$. The correction has been similarly negligible in all subsequent measurements of $G$ until that of Luther and Towler, no less than 22 centuries after Archimedes.

Vietnamska Z,

16000 Prague 6, Czechoslovakia

\section{Relief in microscopy}

SIR - The illumination systems and techniques involved in the production of the photomicrographic image are complex and radically different from those encountered in everyday life, and ambiguities and misinterpretations can arise if the brain interprets the apparently simple image in the same way as it would interpret an ordinary macroscopic photograph.

One of the most common ambiguities is illustrated on the right. The image is an electron micrograph of a metallic replica of a freeze cleaved specimen which on the left is shown in its normal orientation while the right hand photograph has been inverted.

The inversion results in a reversal of relief from cameo to intaglio. This reversal of apparent relief is simply explained by the direction of the shadows. In everyday life, shadows project downwards from objects since most lighting is overhead, so when a photograph presents shadows projecting upwards from an object the brain wrongly interprets an upward shadow from the rim of a depression as a downward shadow from a raised structure and this results in an incorrect perception of surface relief.

The misrepresentation of surface relief is not the only source of ambiguity (D.J.C., Microscopy 34, 533-552; 1983) and microscopists should be aware of the optical illusions which can occur in photomicrographs.

D. J. COOK

School of Pharmacy,

Portsmouth Polytechnic, Portsmouth POI 2DZ, UK

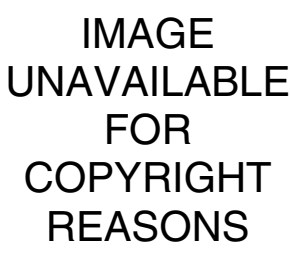

A replica of a fractured surface. The correctly oriented image on the left shows a series of channels (a) which in the inverted photograph on the right appear as ridges. Other relief features show a similar relief reversal. (Reproduced with kind permission of The Upjohn Co., Kalamazoo, Michigan.) 